\title{
Dynamic Delayed Controllers for Unstable Recycling Systems with Time Delays
}

\author{
R. J. VAZQUEZ GUERRA, J. F. MARQUEZ RUBIO, \\ B. del-MURO-CUÉLLAR, G. I. DUCHÉN SÁNCHEZ \\ Sección de Estudios de Posgrado e Investigación, \\ ESIME Unidad Culhuacán, Instituto Politécnico Nacional, \\ 1000, Av. Santa Ana, Coyoacán, 04430, D. F. México \\ rjjvg@yahoo.com.mx,jfcomr23@yahoo.com.mx, bdelmuro@yahoo.com,gduchen@ipn.mx
}

\begin{abstract}
In this work the problem of stabilization and control for recycling system is considered. Such class of systems is characterized by possessing two main paths named through this work as the direct (feedforward) and the recycling feedback) paths. This work considers recycling systems composed by a system of order $n$ with one unstable pole at the direct path and a stable system of order $m$ in the recycling path, both with different time delays. Two different dynamic delayed controllers are proposed in order to achieve a stable behavior of the closed-loop system. Stability conditions for the existence of these controllers are stated. The problems of step tracking and reject step disturbances are also addressed.
\end{abstract}

Keywords: Recycling system; Unstable processes; Time delay; Stabilization.

\section{Introduction}

Recycling systems enable recovering energy and matter in a process. Such class of systems are commonly found in chemical industry for reducing the waste of reactants and hence, the cost of a reaction. Recycling systems are indeed closed loop feedback systems before being controlled. The term recycling is commonly used in process control literature in order to avoid confusion with single (not recycling) systems in closed loop (or feedback) with the controller. For instance a typical plant formed by reactor/separator process, where reactants are recycled back to the reactor $[12,19]$. Another example is a continuous stirred tank reactor (CSTR), where the output stream of the reactor is sent through a separation process, then, the unreacted reactants are returned into the CSTR through pipes. The presence of recycle streams introduces either positive or negative feedback structures into the system that can give rise to some undesirable effects [15]. An example of a recycling system is the recycle of the energy developed by an exothermic reaction in an adiabatic plug flow reactor for feed preheating. Instability could occur due to the exponential increase in the reaction rate with the temperature if it is not properly controlled [4]. Some works have studied the effects of the recycling systems, for instance, Luyben studied the effects of the recycle paths on dynamics process and their implications to plantwide control $[10,11]$. Scali and Ferrari discussed the robust control for recycling plants and proposed the concept of recycle compensation [16]. Similar approaches were extended by Lakshminarayanan and Takada [7], and Kwok et. $\mathrm{Al}$ [6]. In practice, it is quite common to ignore the recycle delay and use standard ordinary differential equation models. However, there has never been a theoretical explanation why this consideration is valid [9]. In fact, these references consider precisely simple ordinary differential equations for the analysis. It should be highlighted that the control problem becomes more complex when time delays in both, the direct and the recycling paths are present. In this situation, the transfer function of the total recycling system has a delay term in the numerator and additionally a second delay term in the characteristic equation. Then, the use of control structures for systems with a single time delay in the numerator, i.e., in the direct path, cannot be directly applied. For instance, the classical Smith Predictor deals with stable systems containing a single delay term in the transfer function. By another way, with an approach inspired in the Smith Predictor strategy, Márquez et. $\mathrm{al}^{14}$ have proposed a control methodology for recycling systems but restricted to unstable first order system at the direct path. This methodology is based on an estimated feedback taken from an observer-predictor.

This work considers the control problem of recycling systems composed by a system with $n$ stable and one unstable poles at the direct 
path and $m$ stable poles in the recycling path, both trajectories containing time delays. The key idea in our proposal is to use a delayed control feedback in order to establish the conditions to guarantee the closed loop stability. The idea of introducing a delay term into the controller has been previously used in the literature. For instance Abdallah et al. [1] consider a delayed term into controller for vibration mitigation. The dynamic delayed controllers here proposed do not only tackle a more general family of plants than the considered in Márquez et. $\mathrm{Al}$ [14], they are also easier to implement considering that this new proposal do not use any observer scheme to design the control law. In fact, the main idea of this work is to explore the simplest way to stabilize the recycling process with time delays and internal instability. The conditions for the existence of the proposed dynamic controllers are stated in terms of the poles position and the delays magnitude. It is important to remark that the problem of stabilization and control of delayed unstable plants even without a recycling path is not completely solved. For instance, recent works [8,17] deal with the stabilization and control of delayed systems with only one unstable pole. Also, note that the stability results for systems with delay in the direct input-output path (see Lee [8], Silva [17], Wang [18], for instance) cannot be applied to the class of system here considered, where two delay terms of different magnitude are obtained in the characteristic equation when the control loop is closed.

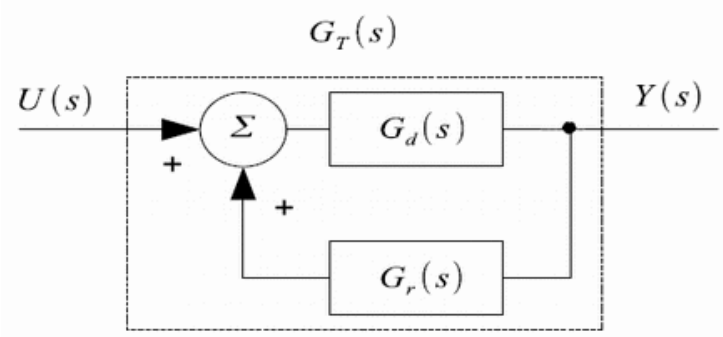

Figure 1. Recycling system

\section{Problem Statement}

Consider the class of recycling system shown in Figure 1, which can be described as

$$
Y(s)=\left[\begin{array}{ll}
G_{d}(s) & G_{d}(s) G_{r}(s)
\end{array}\right]\left[\begin{array}{c}
U(s) \\
Y(s)
\end{array}\right],
$$

with

$$
\begin{aligned}
& G_{d}(s)=\frac{N_{1}}{D_{1}(s)} e^{-\tau_{1} s}, \\
& G_{r}(s)=\frac{N_{2}}{D_{2}(s)} e^{-\tau_{2} s},
\end{aligned}
$$

where $U(s)$ is the process input and $Y(s)$ is the process output. $G_{d}(s)$ and $G_{r}(s)$ are transfer functions of the direct (feedforward) and the recycling (feedback) paths, respectively; $\tau_{1}, \tau_{2} \geq 0$ are the time delays associated to $G_{d}(s)$ and $G_{r}(s) . N_{1}$ and $N_{2}$ are constants; $D_{1}(s)$ and $D_{2}(s)$ are polynomials on the complex variable $s$. The open-loop transfer function of the recycling system (1) is given by

$$
\begin{aligned}
G_{T}(s) & =\frac{Y(s)}{U(s)}= \\
& =\frac{N_{1} D_{2}(s) e^{-\tau_{1} s}}{D_{1}(s) D_{2}(s)-N_{1} N_{2} e^{-\left(\tau_{1}+\tau_{2}\right) s}},
\end{aligned}
$$

Now, for this class of systems (2), a traditional output feedback control of the form

$$
U(s)=C(s)[R(s)-Y(s)]
$$

yields a closed-loop system given by

$$
\begin{aligned}
& \frac{Y(s)}{R(s)}= \\
& =\frac{C(s) N_{1} D_{2}(s) e^{-\tau_{1} s}}{D_{1}(s) D_{2}(s)+\left[C(s) N_{1} D_{2}(s)-N_{1} N_{2} e^{-\tau_{2} s}\right] e^{-\tau_{1} s}}
\end{aligned}
$$

Then the characteristic quasipolynomial is obtained

$$
\begin{aligned}
E(s) & =D_{1}(s) D_{2}(s)-N_{1} N_{2} e^{-\left(\tau_{1}+\tau_{2}\right) s}+ \\
& +C(s) N_{1} D_{2}(s) e^{-\tau_{1} s}
\end{aligned}
$$

It is clear that the characteristic equation $E(s)=0$ has two transcendental terms $e^{-\left(\tau_{1}+\tau_{2}\right) s}$ and $e^{-\tau_{1} s}$. These transcendental terms induce an infinite number of poles [2]. Moreover, a characteristic equation with two different time delays is more complicated to analyze than a characteristic equation with a single time delay [5] and the stability results for systems with a single delay term in the direct input - output path [Lee [8], Silva [17], for instance] cannot be applied. Accordingly, recycling systems are challenging problems for control analysis and design. This work proposes two dynamic delayed controllers that use an output feedback in order to stabilize a class of recycling system. 


\section{Main Results}

Let us consider the class of recycling systems studied in this work, and characterized by

$$
\begin{aligned}
G_{d}(s) & =\frac{N_{1}}{D_{1}(s)} e^{-\tau_{1} s}= \\
& =\frac{N_{1}}{(s-a)\left(s+b_{d 1}\right) \ldots\left(s+b_{d n}\right)} e^{-\tau_{1} s}, \\
G_{r}(s) & =\frac{N_{2}}{D_{2}(s)} e^{-\tau_{2} s}= \\
& =\frac{N_{2}}{\left(s+b_{r 1}\right) \ldots\left(s+b_{r m}\right)} e^{-\tau_{2} s},
\end{aligned}
$$

where $a, b_{d i}, b_{r l} \in R$, with $a, b_{d i}, b_{r l}>0$, $\forall i=1,2, \ldots, n$ and $\forall l=1,2, \ldots, m$. In this case $G_{d}(s)$ is considered unstable and $G_{r}(s)$ is Hurwitz stable.

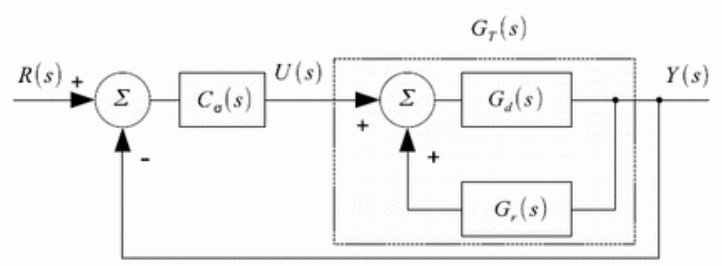

Figure 2. Dynamic delayed $C_{\sigma}(s)$ controller in closed-loop with a recycling system

\subsection{Proposed dynamic delayed controllers}

First, consider the recycling system of Figure 1 with $G_{d}(s)$ and $G_{r}(s)$ and given by (3), the openloop transfer function $G_{T}(s)$ defined in (2) and a new controller $G_{\sigma}(s)$,

$$
U(s)=C_{\mathrm{o}}(s)[R(s)-Y(s)],
$$

with $\sigma=1,2$ and where $R(s)$ is an input reference. Then, the closed-loop transfer function in Figure 2 is

$$
\begin{aligned}
& \frac{Y(s)}{R(s)}= \\
& =\frac{C_{\sigma}(s) N_{1} D_{2}(s) e^{-\tau_{1} s}}{D_{1}(s) D_{2}(s)+\left[C_{\sigma}(s) N_{1} D_{2}(s)-N_{1} N_{2} e^{-\tau_{2} s}\right] e^{-\tau_{1} s}}
\end{aligned}
$$

Then, in what follows the main results of this work are presented.

Theorem 1 Consider the open-loop recycling system given by (2) and the control scheme shown in Figure 2. Then there exists a dynamic $C_{1}(s)$ controller given by

$$
C_{1}(s)=\frac{K_{P}}{D_{2}(s)} e^{-\tau_{2} s}
$$

such that the closed-loop system (4) (with $\sigma=1$ ) is stable if and only if

$\phi<\frac{1}{a}-\sum_{i=1}^{m} \frac{1}{b_{d i}}-\sum_{l=1}^{m} \frac{1}{b_{r l}}$

where $\phi=\tau_{1}+\tau_{2}$.

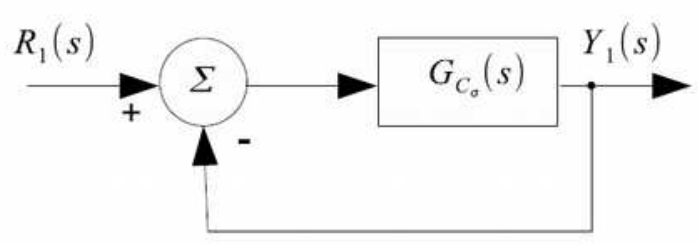

Figure 3. System $G_{C \sigma}(s)$

Proof. Consider $K_{P}=\frac{\overline{K_{P}}+N_{1} N_{2}}{N_{1}}$ and substituting $C_{1}(s)$ into the characteristic equation in (4), yields

$$
E_{1}(s)=D_{1}(s) D_{2}(s)+\overline{K_{p}} e^{-\phi s}=0
$$

Note that the time delay in the $C_{1}(s)$ controller contributes to obtain the characteristic equation (7). The stability properties of the characteristic equation given by (7) are obtained by considering the following auxiliary system shown in Figure 3 with $\sigma=1$, where

$$
G_{C_{1}}(s)=\frac{\overline{K_{P}} e^{-\phi s}}{D_{1}(s) D_{2}(s)}
$$

It can be seen that closed-loop characteristic equation of the system shown in Figure 3, $Y_{1}(s) / R_{1}(s)$, is equivalent to the characteristic equation (7). In such case, the free control parameter is $\bar{K}_{P}$. In what follows a frequency domain analysis with the approach proposed in Lee [8] is used in order to obtain the stability conditions of the auxiliary closedloop system.

(Necessity) The open-loop frequency response of the system (8) is given by

$$
\begin{aligned}
& G_{C_{1}}(j \omega)= \\
& =\frac{\frac{\overline{K_{P}}}{a b_{T}} e^{-\phi j \omega}}{\left(j\left(\frac{1}{a}\right) \omega-1\right) \prod_{i=1}^{n}\left(j\left(\frac{1}{b_{d i}}\right) \omega+1\right) \prod_{l=1}^{m}\left(j\left(\frac{1}{b_{r l}}\right) \omega+1\right)}
\end{aligned}
$$

where $b_{T}=\prod_{i=1}^{n} b_{d i} \prod_{l=1}^{m} b_{r l}$.

Then, the magnitude is given as 


$$
\begin{aligned}
& M_{G_{C_{l}}}(j \omega)= \\
& =\frac{\frac{\bar{K}_{P}}{a b_{T}}}{\sqrt{\left(1+\left(\frac{1}{a}\right)^{2} \omega^{2}\right) \prod_{i=1}^{n}\left(1+\left(\frac{1}{b_{d i}}\right)^{2} \omega^{2}\right) \prod_{l=1}^{m}\left(1+\left(\frac{1}{b_{r l}}\right)^{2} \omega^{2}\right)}}
\end{aligned}
$$

The phase is expressed as

$$
\begin{aligned}
& \angle G_{C_{1}}(j \omega)=-\pi-\phi \omega+\arctan \left(\frac{1}{a} \omega\right)- \\
& -\sum_{i=1}^{n} \arctan \left(\frac{1}{b_{d i}} \omega\right)-\sum_{l=1}^{m} \arctan \left(\frac{1}{b_{r l}} \omega\right)
\end{aligned}
$$

The Nyquist stability criterion establishes that the closed-loop system shown in Figure 3 is stable iff $0=N+P$, with $P$ the number of poles of the transfer function $G_{C_{1}}$ in the right half plane and $N$ the number of counterclockwise rotations to the point $(-1,0)$ in the Nyquist diagram. In this case, $P=1$ due to $G_{C 1}$ has one unstable pole, therefore one counterclockwise rotation is required in order to assure closed-loop stability. From (10), we see that $\angle G_{C_{1}}(0)=-\pi$, then in order to obtain a counterclockwise rotation to the point $(-1,0)$, the phase expression should satisfy $\angle G_{C_{1}}(j \omega)>-\pi$ for $\omega \approx 0$. Therefore, this last condition can be assured by considering $\left.\frac{d}{d \omega}\left(\angle G_{C_{1}}(j \omega)\right)\right|_{\omega=0}>0$. Since

$$
\begin{gathered}
\left.\frac{d}{d \omega}\left(\angle G_{C_{1}}(j \omega)\right)\right|_{\omega=0}= \\
-\phi+\frac{1}{a}-\sum_{i=1}^{n} \frac{1}{b_{d i}}-\sum_{l=1}^{m} \frac{1}{b_{r l}}>0
\end{gathered}
$$

the following stability condition is obtained,

$$
\phi<\frac{1}{a}-\sum_{i=1}^{n} \frac{1}{b_{d i}}-\sum_{l=1}^{m} \frac{1}{b_{r l}}
$$

(Sufficiency) Consider that the condition $\phi<\frac{1}{a}-\sum_{i=1}^{n} \frac{1}{b_{d i}}-\sum_{l=1}^{m} \frac{1}{b_{r l}} \quad$ is satisfied, then $\left.\frac{d}{d \omega}\left(\angle G_{C_{1}}(j \omega)\right)\right|_{\omega=0}>0$. This is, $\angle G_{C_{1}}(j \omega)$ is an increasing function for frequencies $\omega \approx 0$. Therefore an adequate $\overline{K_{P}}$ produces a counterclockwise rotation to the point $(-1,0)$ obtaining the closed-loop stability.

In what follows the set of stabilizing gains $\overline{K_{P}}$ is obtained. For this purpose, the phase crossover frequency $\omega_{c}$ is given by

$$
\begin{gathered}
-\phi \omega_{c}+\arctan \left(\frac{1}{a} \omega_{c}\right)-\sum_{i=1}^{n} \arctan \left(\frac{1}{b_{d i}} \omega_{c}\right)- \\
-\sum_{l=1}^{m} \arctan \left(\frac{1}{b_{r l}} \omega_{c}\right)=0
\end{gathered}
$$

where $\omega_{c}>0$. Also notice that (11) is derived from $\angle G_{C_{1}}\left(\omega_{c}\right)=-180$. For systems where the phase decreases with the frequency (as in the case of $G_{c 1}$ ) such that its phase expression $\left(\angle G_{c 1}\right)$ crosses $-180^{\circ}$ only once from above at frequency $\omega_{c}$, one may use the stability condition $\left|G_{c 1}\left(\omega_{c}\right)\right|<1$, which gives as result, the set of stabilizing gains $\overline{K_{P}}$

$$
a b_{T}<\overline{K_{P}}<a b_{T} M
$$

where

$$
\begin{aligned}
& M=\sqrt{\left(1+\left(\frac{1}{a}\right)^{2} \omega_{c}^{2}\right) \prod_{i=1}^{n}\left(1+\left(\frac{1}{b_{d i}}\right)^{2} \omega_{c}^{2}\right) \prod_{l=1}^{m}\left(1+\left(\frac{1}{b_{r l}}\right)^{2} \omega_{c}^{2}\right)} \\
& \text { and } b_{T}=\prod_{i=1}^{n} b_{d i} \prod_{l=1}^{m} b_{r l} .
\end{aligned}
$$

Remark 1 The $C_{1}(s)$ controller is not able to reject step disturbances neither to track step references. In order to take into account the mentioned issues, in the next paragraphs a second dynamic controller with integral factor is proposed.

Theorem 2 Consider the open-loop recycling system given by (2), and the control scheme shown in Figure 2. Then there exists a dynamic $\mathrm{C}_{2}(s)$ controller given as

$$
C_{2}(s)=\frac{K_{P}(s+\bar{\alpha})}{s D_{2}(s)} e^{-\tau_{2} s}
$$

such that the closed-loop system (4) (with $\sigma=2$ ) is stable if and only if

$$
\phi<\frac{1}{a}-\sum_{i=1}^{n} \frac{1}{b_{d i}}-\sum_{l=1}^{m} \frac{1}{b_{r l}}
$$

where $\phi=\tau_{1}+\tau_{2}$.

Proof. Consider $\quad K_{P}=\frac{\overline{K_{P}}+N_{1} N_{2}}{N_{1}}$, $\bar{\alpha}=\frac{\overline{K_{P}} \alpha}{\overline{K_{P}}+N_{1} N_{2}}$ and substituting $C_{2}(s)$ into the characteristic equation in (4), leads to

$$
E_{2}(s)=D_{1}(s) D_{2}(2)+\frac{\bar{K}_{P}(s+\alpha)}{s} e^{-\phi \alpha}=0
$$

Now the stability conditions of the characteristic equation (15), can be obtained 
from the auxiliary system shown in Figure 3 with $\sigma=2$, where

$$
G_{C_{2}}(s)=\frac{\overline{K_{P}}(s+\alpha) e^{-\phi s}}{s D_{1}(s) D_{2}(s)}
$$

Notice that the characteristic equation (15) and the closed-loop characteristic equation of the auxiliary system shown in Figure 3 are similar. Therefore the stability properties of the auxiliary system are obtained by using the frequency domain analysis with a similar approach as used in Lee [8].

(Necessity) The open-loop frequency response of the system (16) is given by

$$
\begin{aligned}
& G_{C_{2}}(j \omega)=\frac{\overline{K_{P}}}{a b_{T}}\left(j \frac{1}{\alpha} \omega+1\right) e^{\phi j \omega} \\
& =\frac{1}{j \frac{1}{\alpha} \omega\left(j\left(\frac{1}{\alpha}\right) \omega-1\right) \prod_{i=1}^{n}\left(j\left(\frac{1}{b_{d i}}\right) \omega+1\right) \prod_{l=1}^{m}\left(j\left(\frac{1}{b_{r l}}\right) \omega+1\right)} \\
& \text { with } b_{T}=\prod_{i=1}^{n} b_{d i} \prod_{l=1}^{m} b_{r l} .
\end{aligned}
$$

Then, the magnitude $M_{G_{C_{1}}(j \omega)}$ and phase $\angle G_{C_{2}}(j \omega)$ are obtained as (17) and (18).

Notice that the expressions (17) and (18) of the system (16) are equivalent to the expression (9) and (10) of the system (8) when $\alpha=0$.

Therefore, there exist a sufficiently small $\alpha$ gain such that the stability condition of the system (8) can be applied to the system (16), which produces the stability condition

$$
\phi<\frac{1}{a}-\sum_{i=1}^{n} \frac{1}{b_{d i}}-\sum_{l=1}^{m} \frac{1}{b_{r l}}
$$

(Sufficiency) Consider that the condition $\phi<\frac{1}{a}-\sum_{i=1}^{n} \frac{1}{b_{d i}}-\sum_{l=1}^{m} \frac{1}{b_{r l}}$ is satisfied, and that there exist a sufficiently small $\alpha$ gain such that

$$
\left.\frac{d}{d \omega}\left(\angle G_{C_{2}}(j \omega)\right)\right|_{\omega}=0>0 \text {. Then } \angle G_{C_{2}}(j \omega)
$$

is an increasing function for low frequencies.

And an adequate $\overline{K_{P}}$ produces a counterclockwise rotation to the point $(-1,0)$ ensuring the stability.

In what follows the parameters of the controller provided by Theorem 2 are obtained. First, $\alpha$ is selected such that the following equation is solved

$$
\begin{aligned}
& -\phi \omega_{c}+\arctan \left(\frac{1}{a} \omega_{c}\right)-\arctan \left(\frac{\alpha}{\omega_{c}}\right)- \\
& -\sum_{i=1}^{n} \arctan \left(\frac{1}{b_{d i}} \omega_{c}\right)-\sum_{l=1}^{m} \arctan \left(\frac{1}{b_{r l}} \omega_{c}\right)=0
\end{aligned}
$$

We define $\omega_{c 1}>0$ and $\omega_{c 2}>0$ as the first two phase crossover frequencies of (19). Also notice that (19) is derived from $\angle G_{C 2}\left(\omega_{c}\right)=-180$. Then, we use the stability condition $\left|G_{C 2}\left(\omega_{c}\right)\right|<1$, which gives as result the set of stabilizing gains $\overline{K_{P}}$.

$$
\frac{a b_{T}}{\alpha} M_{1}<\overline{K_{P}}<\frac{a b_{T}}{\alpha} M_{2}
$$

$$
M_{g}=\sqrt{\frac{\left(1+\left(\frac{1}{a}\right)^{2} \omega_{c g}^{2}\right) \prod_{i=1}^{n}\left(1+\left(\frac{1}{b_{d i}}\right)^{2} \omega_{c g}^{2}\right) \prod_{l=1}^{m}\left(1+\left(\frac{1}{b_{r l}}\right)^{2} \omega_{c g}^{2}\right)}{1+\left(\frac{\alpha}{\omega_{c g}}\right)^{2}}}
$$

with $g=1,2$ and where $b_{T}=\prod_{i=1}^{n} b_{d i} \prod_{l=1}^{m} b_{r l}$.

Remark 2 The proposed $C_{2}(s)$ controller satisfies, the properties of step tracking reference and rejecting step disturbance in the closed-loop system. This can be easily verified by using the proposed controller and applying the final value theorem.

\section{Robustness: Uncertainties in the Parameters and in the Delay Terms}

In the preceding developments, a control strategy has been presented under the assumption of a complete knowledge of the actual process. In practice, it is desired to get a control strategy that provides stability conditions with respect to model uncertainties. In what follows, two different approaches are used in order to analyze robustness issues with respect

$$
\begin{aligned}
& M_{G_{c_{2}}(j \omega)}=\frac{\overline{K_{P}}}{a b_{T}} \sqrt{\frac{1+\left(\frac{\alpha}{\omega}\right)^{2}}{\omega^{2}\left(1+\left(\frac{1}{\alpha}\right)^{2} \omega^{2}\right) \prod_{i=1}^{n}\left(1+\left(\frac{1}{b_{d i}}\right)^{2} \omega^{2}\right) \prod_{l=1}^{m}\left(1+\left(\frac{1}{b_{r l}}\right)^{2} \omega^{2}\right)}} \\
& \angle G_{C_{2}}(j \omega)=-\pi-\phi \omega+\arctan \left(\frac{1}{\alpha} \omega\right)-\arctan \left(\frac{\alpha}{\omega}\right)-\sum_{i=1}^{n} \arctan \left(\frac{1}{b_{d i}} \omega\right)-\sum_{l=1}^{m} \arctan \left(\frac{1}{b_{r l}} \omega\right)
\end{aligned}
$$


to the proposed control strategies applied to recycling systems. The first presented approach addresses the problem of stability when uncertainties with respect to the plant parameters are considered. Subsequently a second approach allows analysing the control strategies robustness with respect to uncertainties in the time delays. Additionally, both analysis strategies can be used simultaneously.

\subsection{Parameters uncertainties}

The first step towards the robustness analysis consists in taking into account parametric uncertainties in the recycling system. Therefore, it is assumed that there are no uncertainties in the time delays. For simplicity in the notation, we dropped the argument $s$ in the polynomials. Consider now a general representation of the proposed controllers $C_{1}(s)$ and $C_{2}(s)$ as

$$
C_{\sigma}=\frac{N_{C}}{D_{C}} e^{-\tau_{c} s}
$$

where $N_{C}$ represents the numerator, $D_{C}$ is the denominator of the controller and $e^{-\tau_{c} s}$ is the delay term. Since robustness analysis with respect to uncertainties in the plant parameters is considered, the plant with uncertain parameters is defined as

$$
\bar{G}_{T}(s)=\frac{\bar{G}_{d}(s)}{1-\bar{G}_{d}(s) \bar{G}_{r}}
$$

with

$$
\begin{aligned}
\bar{G}_{d}(s) & =\frac{\bar{N}_{1}}{\bar{D}_{1}} e^{-\tau_{1} s}= \\
& =\frac{N_{1}+N_{1}^{0}}{\left(s-a+a^{0}\right)\left(s+b_{d 1}+b_{d 1}^{0}\right) \ldots\left(s+b_{d n}+b_{d n}^{0}\right)} e^{-\tau_{1} s}, \\
\bar{G}_{r} & =\bar{N}_{2} \bar{D}_{2} e^{-\tau_{2} s}= \\
= & \frac{N_{2}+N_{2}^{0}}{\left(s+b_{r 1}+b_{r 1}^{0}\right) \ldots\left(s+b_{r m}+b_{r m}^{0}\right)} e^{-\tau_{2} s}
\end{aligned}
$$

where $N_{1}^{0}, a^{0}, b_{d 1}^{0}, \ldots, b_{d n}^{0}, N_{2}^{0}, b_{r 1}^{0}, \ldots$, $b_{r m}^{0}$ are the uncertainties on the parameters plant. Notice that when

$$
N_{1}^{0}=a^{0}=b_{d 1}^{0}=\ldots=b_{d n}^{0}=N_{2}^{0}=b_{r 1}^{0}=\ldots=b_{r m}^{0}=0
$$

the system (22) becomes the nominal process (2). In this subsection, it is assumed $\tau_{c}=\tau_{2}$ and no uncertainties in the time delays. Therefore, consider the proposed control structure shown in Figure 2 with the uncertainty parameters plant $\bar{G}_{T}(s)$ given by (22) and the general control given by (21), then the closed-loop characteristic equation

$$
D_{c} \bar{D}_{1} \bar{D}_{2}+\left(N_{c} \bar{D}_{2}-D_{c} \bar{N}_{2}\right) \bar{N}_{1} e^{-\left|\tau_{1}+\tau_{2}\right| s}=0
$$

has a single time delay term $\left(e_{-\left(\tau_{1}+\tau_{2} \mid s\right.}\right)$, which allows analyzing its stability by means of a traditional Nyquist criteria.

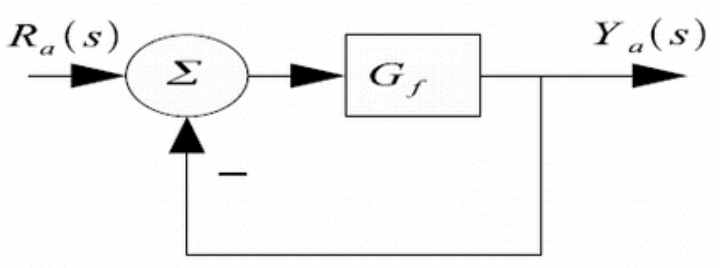

Figure 4. Auxiliary system

This can be done by considering an auxiliary system shown in Figure 4, where

$$
G_{f}=\frac{N_{c} \bar{N}_{1} \bar{D}_{2}-D_{c} \bar{N}_{1} \bar{N}_{2}}{D_{c} \bar{D}_{1} \bar{D}_{2}} e^{-\left(\tau_{1}+\tau_{2}\right) s}
$$

Note that the closed-loop characteristic equation of the auxiliary system shown in Figure 4 is the same that the characteristic equation given by (23). In this way, the stability Nyquist criteria can be applied to the auxiliary system shown in Figure 4 to obtain the stability results of the characteristic equation (23). Also notice that $G_{f}$ includes the parameters of the open-loop system as well as the control parameters. It should be pointed out that we have made such transformation of the system in order to apply the Nyquist stability criterion in a traditional way, which states that when the loop is closed, the system will be stable iff $0=N+P$, with $P$ the number of poles of the transfer $G_{f}$ in the right half plane and $\mathrm{N}$ the number of clockwise round trips to the point -1 ( $\mathrm{N}$ negative in counterclockwise) in the Nyquist diagram. Then, we can verify that when

$$
N_{1}^{0}=a^{0}=b_{d 1}^{0}=\ldots=b_{d n}^{0}=N_{2}^{0}=b_{r 1}^{0}=\ldots=b_{r m}^{0}=0
$$

(nominal system case), the Nyquist diagram of the $G_{f}$ satisfies the mentioned stability conditions. Moreover we can set uncertainties on the open loop system i.e., $N_{1}^{0}, a^{0}, b_{d 1}^{0}, \ldots, b_{d n}^{0}, N_{2}^{0}, b_{r 1}^{0}, \ldots, b_{r m}^{0} \quad$ different from zero and verify if the Nyquist diagram satisfies the stability properties. This last analysis is just the key to analyze the robustness issue of the proposed control 
strategy, and will be illustrated in the section of Simulation Results.

\subsection{Time delay uncertainties}

Here it will be shown that the results presented in $\mathrm{Gu}$ et. $\mathrm{al}^{5}$ can be used in order to analyze the robustness properties of the control strategy addressed in this work with respect to uncertainties in the time-delay. In particular, because the time delay used in the controller may be different from one associated with the plant. With this aim, consider a characteristic quasipolynomial of the form

$$
p(s)=p_{0}(s)+p_{1}(s) e^{-\tau_{a} s}+p_{2}(s) e^{-\tau_{b} s}=0
$$

where its stability properties will be established as a function of the time-delays $\tau_{a}$ and $\tau_{b}$. Following [5] it is possible to give a general framework for our particular case. Let $T$ denote the set of all points $\left(\tau_{a}, \tau_{b}\right) \in R_{+}^{2}$ (where $R_{+}^{2}$ denotes the set of vectors in $R^{2}$ with positive elements) such that $p(s)$ has at least one zero on the imaginary axis. Any $\left(\tau_{a}, \tau_{b}\right) \in T$ is known as a crossing point and $T$ is the collection of all stability crossing curves.

Now consider the plant with uncertain time-delays

$$
\hat{G}_{T}(s)=\frac{\hat{G}_{d}(s)}{1-\hat{G}_{d}(s) \hat{G}_{r}(s)}
$$

with

$$
\begin{aligned}
& \hat{G}_{d}(s)=\frac{N_{1}}{D_{1}} e^{-\left(\tau_{1}+\tau_{10} \mid s\right.} \\
& \hat{G}_{r}(s)=\frac{N_{2}}{D_{2}} e^{-\left(\tau_{2}+\tau_{20} \mid s\right.}
\end{aligned}
$$

where $\tau_{10}$ and $\tau_{20}$ are the time-delay uncertainties on the plant. Then, the proposed control structure shown in Figure 2 with the uncertain time-delays plant $\hat{G}_{T}(s)$ given by (26) and the general control given by (21), gives the closed-loop characteristic equation

$$
p_{A}(s)=p_{a}(s)+p_{b}(s) e^{-\tau_{a} s}+p_{c}(s) e^{-\tau_{b} s}=0
$$

with

$$
\begin{aligned}
& p_{a}(s)=D_{c} D_{1} D_{2}, \\
& p_{b}(s)=-D_{c} N_{1} N_{2}, \\
& p_{c}(s)=N_{c} N_{1} D_{2}, \\
& \tau_{a}=\tau_{1}+\tau_{10}+\tau_{2}+\tau_{20}, \\
& \tau_{b}=\tau_{1}+\tau_{10}+\tau_{c}
\end{aligned}
$$

Note that in the nominal case $\tau_{c}=\tau_{2}$, however the characteristic equation (27) allows the analysis of the closed-loop stability when $\tau_{c} \neq \tau_{2}$. It is clear that the characteristic equation (27) has the form of (25), therefore it is possible to identify the region of $\left(\tau_{a}, \tau_{b}\right)$ in $R_{+}^{2}$ such that $p_{A}(s)$ is stable.

\section{Simulation Results}

In this section dynamic delayed controllers are applied to different processes to illustrate the performance.

\subsection{Example 1}

Consider the recycling system of Figure 1 with,

$$
\begin{aligned}
& G_{d}(s)=\frac{N_{1}}{D_{1}}(s) e^{-\tau_{1} s}=\frac{11}{(s-0.4)(s+2)} e^{-0.4 s} \\
& G_{r}(s)=\frac{N_{2}}{D_{2}}(s) e^{-\tau_{2} s}=\frac{3}{(s+5)} e^{-0.5 s}
\end{aligned}
$$

The total time delay is $\phi=0.9$.

\subsubsection{Performance evaluation}

Since the condition (6) of the Theorem 1 is fulfilled, it follows that the recycling system is stabilizable by dynamic $C_{1}(s)$ controller. Computing $\omega_{c}=0.6390$ from (11), and using (12) the range $4<\overline{K_{P}}<7.9785$ is obtained. $\overline{K_{P}}=4.3$ is chosen. From (5) the corresponding dynamic $C_{1}(s)$ controller can be written as,

$$
C_{1}(s)=\frac{K_{P}}{D_{2}}(s) e^{-\tau_{2} s}=\frac{3.3909}{(s+5)} e^{-0.5 s}
$$

Figure 5 shows the stable output response of the system (28) in closed-loop with the dynamic $C_{1}(s)$ controller.

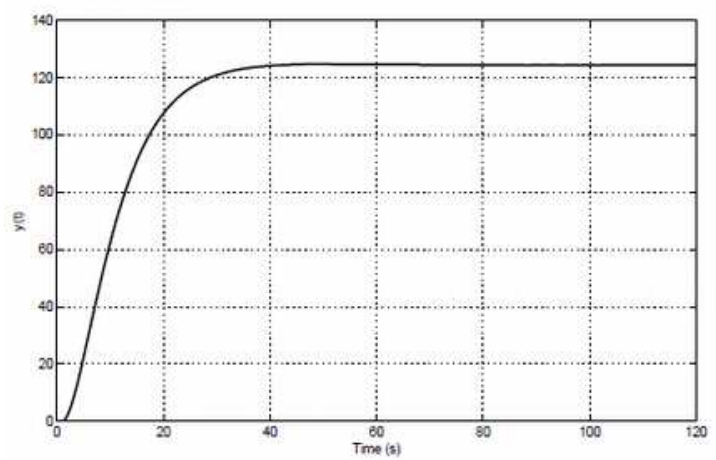

Figure 5. Output response of the system (28) in closed-loop with $\mathrm{C}_{1}(\mathrm{~s})$ controller 
How, the dynamic $C_{2}(s)$ controller provided by Theorem 2 is applied to the recycling system given by (28). Notice that the condition (14) is satisfied, therefore the parameter $\alpha$ is selected as $\alpha=0.03$. From (19), we obtain $\omega_{c 1}(s)=0.2043$ and $\omega_{c 2}(s)=0.5733$. Then $\overline{K_{P}}$ is bounded by $4.4707<\overline{K P}<7.3097$ from (20). The parameter $\overline{K_{P}}=5.3$ is chosen. Then, from (13) the corresponding dynamic controller is,

$$
\begin{aligned}
C_{2}(s) & =\frac{K_{P}(s+\bar{\alpha})}{s D_{2}(s)} e^{-\tau_{2} s}= \\
& =\frac{3.4818(s+0.0042)}{s(s+5)} e^{-0.5 s}
\end{aligned}
$$

Figure 6 shows system output response of the system (28) in closed-loop with the dynamic $C_{2}(s)$ controller, subject to a disturbance steptype acting on the time $t=200 s$ with a magnitude of 0.3 units.

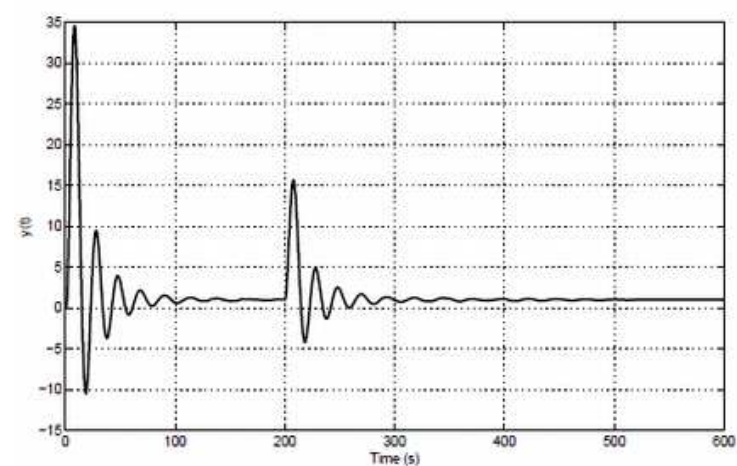

Figure 6. Output response of the system (28) in closed-loop with $\mathrm{C}_{2}(\mathrm{~s})$ controller

\subsubsection{Robustness analysis}

Following the presented results of Robustness with respect to parameters uncertainties, in this Example we analyze the robustness of the closed-loop system when the $C_{1}(s)$ controller is used and an uncertainty on the unstable pole position (parameter $a$ ) is considered. To do this, we build a Nyquist diagram of the system (24) for different values of $a^{0}$, this is shown in Figure 7. From stability Nyquist criteria, the system (24) is stable if and only if the point -1 is encircled in counterclockwise, this due to the unstable pole at open loop of (24). In this way, Figure 7 illustrates that for the nominal case $a^{0}=0$ the system is stable. Then with $a^{0}=0.4$ and $a^{0}=-0.03$ the stability of closed-loop system is at the limit. From this Figure, we can conclude that the closed-loop stability is preserved with an uncertainty of $100 \%$ and $-7.5 \%$ in the parameter $a$.

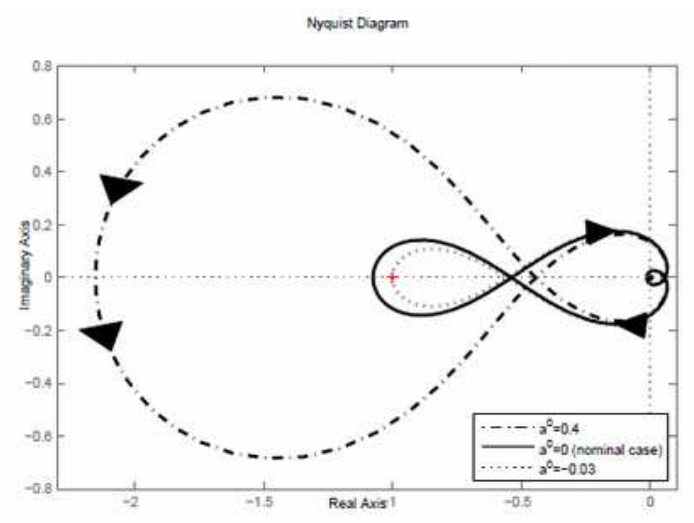

Figure 7. Nyquist diagram for different values of $\mathrm{a}^{0}$ uncertainty.

With a similar development, Figure 8 shows the Nyquist diagram for different values of $b_{r 1}^{0}$, which allows to conclude that the closed-loop stability is preserved with an uncertainty of $-1 \%$ and $+10 \%$ in the parameter $b_{r 1}($ $b_{r 1}=5$ in the nominal case). As we can see, this analysis can be extended for any parameter of the system or the combination of more than one uncertainty.

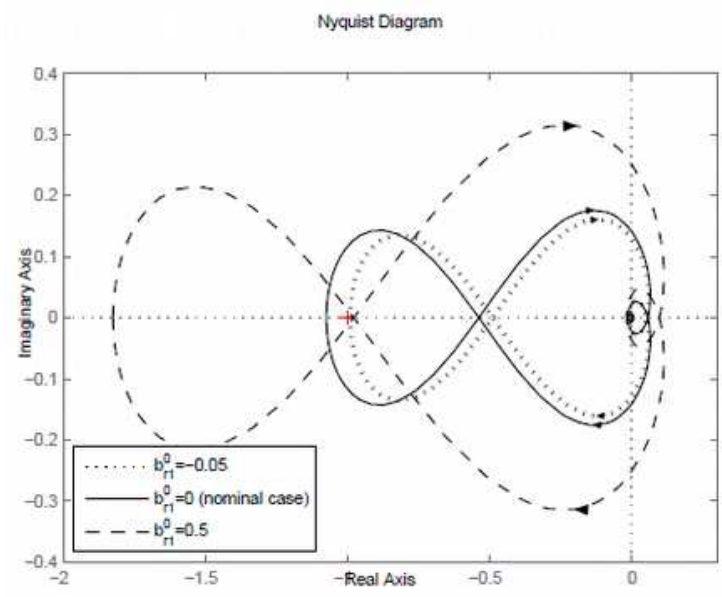

Figure 8. Nyquist diagram for different values of $\mathrm{b}_{\mathrm{rl}}^{0}$ uncertainty.

Now, let us analyze the robustness of the closed-loop system with respect to time delays. Following [5] for each particular example, it is possible to obtain a figure that shows the points $\left(\tau_{a}, \tau_{b}\right)$ such that the characteristic equation (27) has at least one zero on the imaginary axis. Therefore, the figure allows obtaining the minimum and the maximum values of $\tau_{a}$, such that the closed loop of the proposed control strategies remains stable. These values are denoted as $\tau_{a \min }$ and $\tau_{a \max }$. This can be 
also extended to the time delay $\tau_{b}$, with the minimum and the maximum values of $\tau_{b}$, denoted as $\tau_{b \min }$ and $\tau_{b \max }$, respectively. Therefore, our robustness analysis is based on obtaining the minimum and maximum values of $\tau_{10}$ and $\tau_{20}$ such that (27) remains stable, which can be obtained directly from $\tau_{a \min }$, $\tau_{a \max }, \tau_{b \min }$ and $\tau_{b \max }$. Taking into account the characteristic equation (27) and the procedure derived from [5] in Figure 9 it is presented the corresponding region $\left(\tau_{a}, \tau_{b}\right)$ when the $C_{1}(s)$ controller is used. Let us consider an uncertainty $\left(\tau_{20}\right)$ in the time delay $\tau_{2}$. In this way, from Figure 9 we see for $\tau_{b}=0.9$ (this value of $\tau_{b}$ is analyzed due to $\tau_{b}=\tau_{1}+\tau_{c}$ and no uncertainty in the timedelay $\tau_{1}$ ), it is obtained $0.8<\tau_{a}<1.3$ into the stable region. With the observation that $\tau_{a}=\tau_{1}+\tau_{2}+\tau_{20}$, we see that the range of $\tau_{20}$ such that the closed-loop system is stable is $-0.1<\tau_{20}<0.4$. Therefore, with similar analysis, Figure 9 can be also used to obtain the stability range when uncertainties in the time delays $\tau_{1}$ is set.

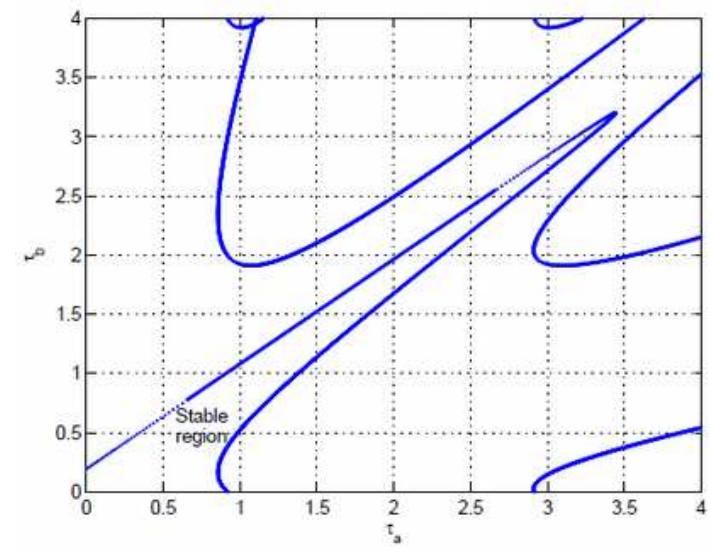

Figure 9. Stability region for $\tau_{\mathrm{a}}$ and $\tau_{\mathrm{b}}$, to $\mathrm{C}_{1}(\mathrm{~s})$ controller, example 1.

Similarly, in Figure 10 is presented the corresponding region $\left(\tau_{a}, \tau_{b}\right)$ when the $C_{2}(s)$ controller is used. In this case, let us also analyze the case where an uncertainty $\left(\tau_{10}\right)$ in the time delay $\tau_{1}$ is considered. We have $\tau_{c}=\tau_{2}=0.5$, therefore $\tau_{a}=0.9+\tau_{10}$ and $\tau_{b}=0.9+\tau_{10}$. From the last equations we can see that the uncertainty $\tau_{10}$ affects $\tau_{a}$ and $\tau_{b}$ at the same time. In this way, in Figure 10, all possibilities of $\left(\tau_{a}, \tau_{b}\right)$ such that the uncertainty is set are presented by means a dashed line. From the dashed line in Figure 10, the maximal values of $\left(\tau_{a}, \tau_{b}\right)$ such that the closed loop system is stable is $(1.1,1.1)$. Now, if we consider that the nominal case is $(0.9$, 0.9 ), we obtain $-0.4<\tau_{10}<0.2$ (the minimum of $\tau_{10}$ is obtained taking into account that $\tau_{1}$ should satisfy $\tau_{1}>0$ ).

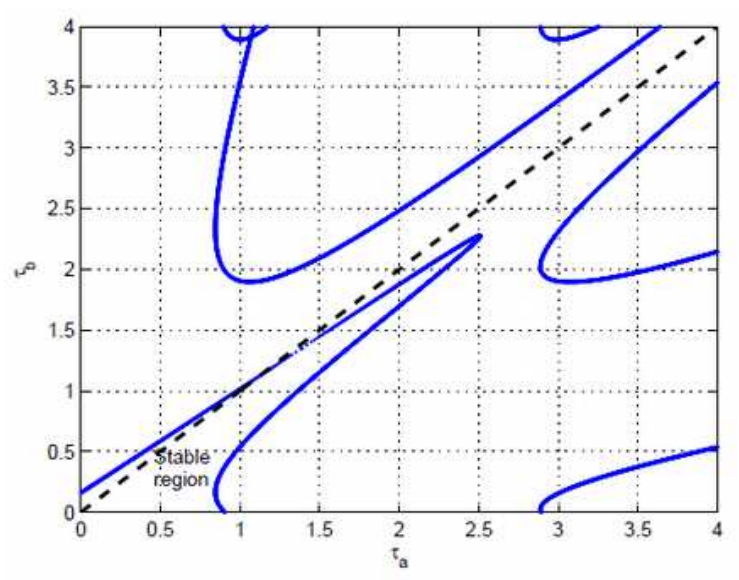

Figure 10.Stability region for $\tau_{\mathrm{a}}$ and $\tau_{\mathrm{b}}$, to $\mathrm{C}_{2}(\mathrm{~s})$ controller, example 1.

In general, Figure 9 and Figure 10 can be used to obtain more than one time-delay uncertainty. Moreover, the two proposed approaches (Nyquist criteria and curves $\left(\tau_{a}, \tau_{b}\right)$ can be also used together in order to consider uncertainties in the parameters and the time delays.

\subsection{Example 2}

Consider the recycling system of Figure 1 with

$$
\begin{aligned}
& G_{d}(s)=\frac{N_{1}}{D_{1}(s)} e^{-\tau_{1} s}=\frac{1}{(s-0.8)(s+6)} e^{-0.6 s} \\
& G_{r}(s)=\frac{N_{2}}{D_{2}(s)} e^{-\tau_{2} s}=\frac{1}{(s+12)} e^{-0.2 s}
\end{aligned}
$$

Since the condition (14) of the Theorem 2 is satisfied, the $C_{2}(s)$ controller can be used for the recycling system (29). Thus, the parameter $a$ is chosen as $a=0.005$, obtaining $\omega_{c 1}(s)=0.1655, \quad \omega_{c 2}(s)=0.6225 \quad$ and therefore $58.82<\overline{K_{P}}<73.47$. For the simulations $\overline{K_{P}}=61$ is selected, from (13) the corresponding dynamic controller is

$$
\begin{aligned}
C_{2}(s) & =\frac{K_{P}(s+\bar{a})}{s D_{2}(s)} e^{-\tau_{2} s}= \\
& =\frac{62(s+0.0049)}{s(s+12)} e^{-0.2 s}
\end{aligned}
$$

In Marquez [13] a scheme observer and a two degree of freedom control [3] have been used. Now with comparison purposes the proposed $C_{2}(s)$ controller given by (30), also can be implemented in two degree of freedom as 


$$
U(s)=R(s) G_{f f}(s)-Y(s) G_{c}(s),
$$

with

$$
\begin{aligned}
& G_{f f}(s)=\frac{K_{P}}{D_{2}}\left(\eta+\frac{\bar{\alpha}}{s}\right) e^{-\tau_{2} s} \\
& G_{c}(s)=\frac{K_{P}}{D_{2}}\left(1+\frac{\bar{\alpha}}{s}\right) e^{-\tau_{2} s}
\end{aligned}
$$

where $\eta$ is a parameter that should be selected from $0<\eta<1$. For the simulations $\eta=0.03$ is chosen. On the other hand, consider the observer/controller methodology proposed by Marquez et al [13]. In this way the observer parameter is used as $k=5$ and a PI controller to the delay-free direct path, given by

$$
\begin{aligned}
& G_{f f}(s)=5.33\left(0.12+\frac{0.0086}{s}\right) \\
& G_{c}(s)=5.33\left(1+\frac{0.0086}{s}\right) .
\end{aligned}
$$

Then, an unitary step reference and a step disturbance $Q(s)$ of magnitude -0.1 acting at $140 \mathrm{sec}$. are considered in order to compare both control strategies. The performance of the output signal is shown in Figure 11. Under these conditions and by considering initial conditions different from zero in the process as well as a process uncertainty at time delay as $\tau_{1}=0$, Figure 12 presents the behavior of the two control strategies. Then, Figure 13 shows the output performance when the position of the process recycling pole $(s=-12)$ has an uncertainty to $s=-5$.

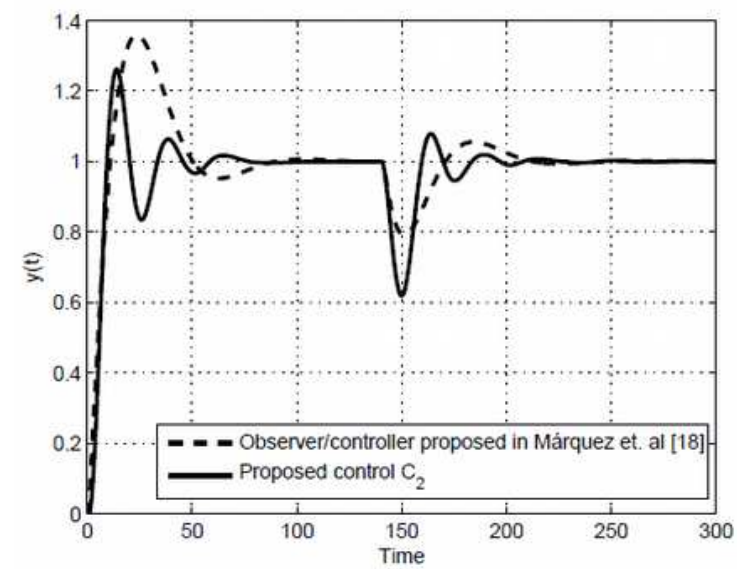

Figure 11. Comparative output response under step disturbance.

Finally, Figure 14 shows the output response when random noise in the output measurement is considered as well as an initial output

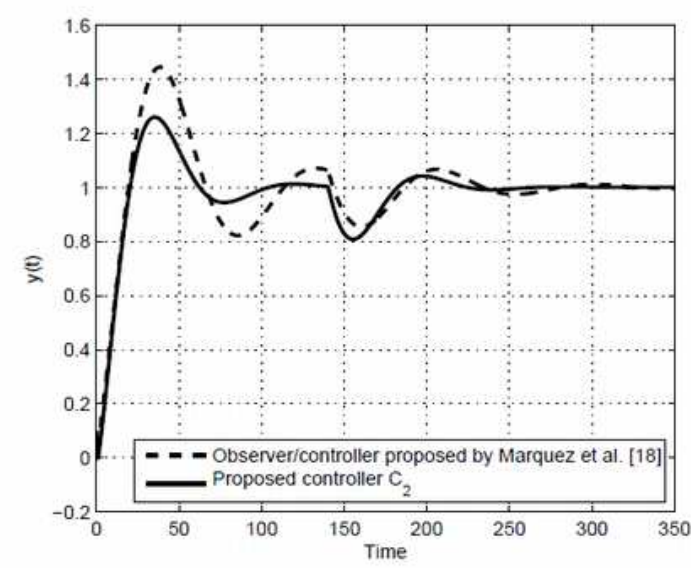

Figure 12. Comparative output response under initial condition different from zero and uncertainty at $\tau_{1}$.

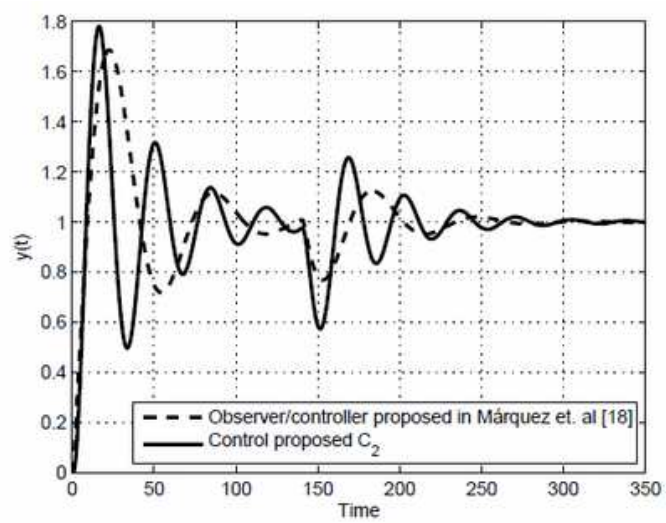

Figure 13. Comparative output response when uncertainty at $\mathrm{D}_{2}(\mathrm{~s})$ is considered.

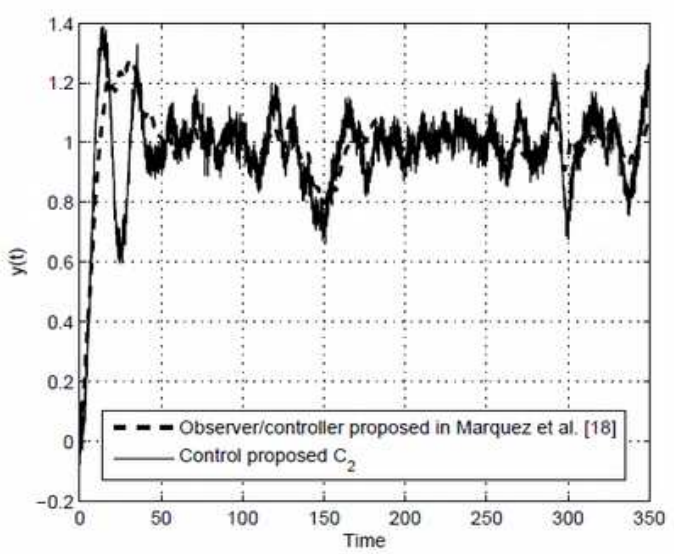

Figure 14.Comparative output response when random noise in the output measurement is considered.

different from zero in the process. As we can see, the observer based controller has a better job in rejecting measurement noise than the proposed controller. In order to assessing quantitatively the output signal performance, Table 1 shows a comparison between the two 
control strategies by using the Integral Time Absolute Error (ITAE) criteria. From Figures 11, 12, 13, 14 and Table 1, we can conclude that similar output response can be obtained by using both control methodologies, however it should be pointed out that the control strategy provided in the present work is simpler than the one presented by Marquez et al [13].

Table 1. ITAE-Criteria

\begin{tabular}{|c|c|c|}
\hline Performance & $\begin{array}{c}\text { Proposed } \\
\text { strategy }\end{array}$ & $\begin{array}{c}\text { Observer } \\
\text { Marquez }^{13}\end{array}$ \\
\hline Figure 11 & 944.7 & 1139 \\
\hline Figure 12 & 1520 & 2607 \\
\hline Figure 13 & 3389 & 2354 \\
\hline Figure 14 & 4024 & 2301 \\
\hline
\end{tabular}

\section{Conclusions}

In this paper two dynamic delayed controllers were proposed in order to stabilize and control recycling system. The recycling system is composed by a system with $n$ stable and one unstable poles at the direct path and $m$ stable poles in the recycling path, both paths with time delay. Conditions for the existence of the corresponding stabilizing controllers are stated. The schemes by dynamic delayed controllers are simple and may be easily implemented. The $C_{2}(s)$ dynamic controller is more complicated than the $C_{1}(s)$ controller but it contains an integral factor that solves the step disturbances rejection problem and the tracking of step references. Numerical simulations are presented in order to illustrate the application of the proposed results. Also, results with respect to the robustness of the control strategy are presented. Then it is illustrated that the proposed strategy allows considerably magnitude of uncertainties.

\section{REFERENCES}

1. ABDALLAH, C., P. DORATO, J. BENITES-READ, R. BYRNE, Delayed Positive Feedback Can Stabilize Oscillatory Systems, American Control Conference, 1993, pp. 3106-3107.

2. ATAY, F. M., (Ed.) Complex Time-Delay Systems: Theory and Applications, Springer: Berlin, 2010.
3. ASTROM, K., T. HAGGLUND, PID Controllers: Theory, Design and Tuning, International Society for Measurement and Control: New York, 1995.

4. BILDEA, C. S., A. C. DIMIAN. Stability and Multiplicity Approach to the Design of Heat- Integrated PFR, AIChE Journal, vol. 44(12), 1998, pp.2703-2712.

5. GU, K., S. I. NICULESCU, J. CHEN, On Stability Crossing Curves for General Systems with Two Delay, Journal of Mathematical Analysis and Application, vol. 311(1), 2005, pp. 231-253.

6. KWOK, K. E., M. CHONG-PING, G. A. DUMONT, Seasonal Model Based Control of Processes with Recycle Dynamics, Industrial Engineering in Chemical Research, vol. 40, 2001, pp. 1633-1640.

7. LAKSHMINARAYANAN, S., H. TAKADA, Empirical Modelling and Control of Processes with Recycle: Some Insights via Case Studies, Chemical Engineering Science, vol. 56, issue 11, 2001, pp. 3327-3340.

8. LEE, S. C., Q. G. WANG, C. XIANG, Stabilization of All-pole Unstable Delay Processes by Simple Controllers, Journal of Process Control, vol. 20(2), 2010, pp. 235-239.

9. LEHMAN, B. Stability of Chemical Reactions in a CSTR with Delayed Recycle Stream, American Control Conference, 1994, pp. 3521-3522.

10. LUYBEN, W. L., Dynamics and Control of Recycle Systems 1. Simple Open-Loop and Closed- Loop Systems, Industrial Engineering in Chemical Research, vol. 32(3), 1993, pp. 466-475.

11. LUYBEN, W. L., Temperature Control of Autorefrigerated Reactor, Journal of Process Control, vol. 9(4), 1999, pp. 301-312.

12. LUYBEN, W. L., B. D. TYREUS, M. L. LUYBEN. Plantwide Process Control, McGraw-Hill: New York, 1998.

13. MARQUEZ, J. F., B. DEL MURO, O. SENAME, Control of Delayed Recycling Systems with an Unstable Pole at Forward Path, American Control Conference. Montréal, Canada, 2012. 
14. MÁRQUEZ, J. F., B. DEL MURO, M. VELASCO, D. CORTÉS, O. SENAME, Control of Delayed Recycling Systems with Unstable First Order Forward Loop, Journal Process Control, vol. 22(4), 2012, pp. 729-737.

15. MORUD, J., S. SKOGESTAD, Dynamic Behavior of Integrated Plants, Journal of Process and Control, vol. 6, iss. 2/3, 1996, pp. 145-156.

16. SCALI, C., F. FERRARI, Performance of Control Systems Based on Recycle Compensators in Integrated Plants, Journal Process Control, vol. 9, iss. 5, 1999, pp. 425-437.
17. SILVA, G. J., A. DATTA, S. P. BHATTACHARYYA, PID Controllers for Time-Delay Systems, Birkhuser: Boston, 2005.

18. WANG, H.,C. VASSEUR, V. KONCARA. CHAMROO, N. CHRISTOV, Sampled Tracking for Delayed Systems Using Two-Time-Scale Sampled-data Controllers, Studies in Informatics and Control, vol. 19(4), 2010, pp. 339-346.

19. WU, K. L., C. C. YU, Reactor / Separator Processes with Recycle-1. Candidate Control Structure for Operability. Computers \& Chemical Engineering vol. 20, iss. 11, 1996, pp. 1291-1316. 